УДК 512.54

\title{
Analysis of an Exact Solution of Problem of the Evaporative Convection (Review). Part II. Three-dimensional Flows
}

\author{
Victoria B. Bekezhanova* \\ Institute of Computational Modeling SB RAS \\ Academgorodok 50/44, Krasnoyarsk, 660036 \\ Institute of Mathematics and Computer Science \\ Siberian Federal University \\ Svobodny, 79, Krasnoyarsk, 660041 \\ Russia \\ Olga N. Goncharova ${ }^{\dagger}$ \\ Institute of Thermophysics SB RAS \\ Lavrentieva, 1, Novosibirsk, 630090 \\ Altai State University \\ Lenina, 61, Barnaul, 656049 \\ Russia \\ Ilia A. Shefer ${ }^{\ddagger}$ \\ Institute of Mathematics and Computer Science \\ Siberian Federal University \\ Svobodny, 79, Krasnoyarsk, 660041 \\ Russia
}

Received 06.07.2017, received in revised form 09.01.2018, accepted 01.02.2018

In the paper the review of exact solutions of the three-dimensional convection problems is presented. The solutions allow one to model the two-layer convective fluid flows with evaporation at the thermocapillary interface.

Keywords: evaporative convection, exact solution, three-dimensional flows, thermocapillary interface. DOI: $10.17516 / 1997-1397-2018-11-3-342-355$.

\section{Introduction}

In the second part of the review we present application of exact solutions of the classical convection equations to description of the two-layer flows in three-dimensional case, among other flows with evaporation. The Ostroumov-Birikh exact solutions of the Oberbeck - Boussinesq equations allow one to study characteristic features of interaction of various mechanisms of the evaporative convection in gravitational field and in weightlessness. The solutions consider the thermodiffusion and diffusive thermal conductivity effects in the gas - vapor phase. In the paper the trajectories of fluid particle motion, temperature fields in a rectangular channel are presented.

\footnotetext{
*vbek@icm.krasn.ru

$\dagger$ gon@math.asu.ru

†ilya.shefer@gmail.com

(c) Siberian Federal University. All rights reserved
} 
These examples of the two-layer convective fluid flows with evaporation are obtained on the basis of new solution of the Navier-Stokes equations in the context of the Oberbeck-Boussinesq approximation. Influence of various factors on the flow structure are discussed.

\section{Three-dimensional flows}

The Ostroumov - Birikh solution can be used to describe two-layer convective flows in threedimensional channel. Generalization of the Ostroumov-Birikh solution in the case of the motion in cylindrical channel of an arbitrary cross-section have been presented [1]. Group analysis of the solution and solvability of corresponding initial boundary value problems have been also studied.

Three-dimensional generalizations of the plane Ostroumov-Birikh solution which describe convection in a rotating circular pipe induced by the centrifugal force and heat flux acting along axes of the duct have been analyzed [2] (see also the references there). In the absence of the transversal gravitational force the stationary solution is expressed in terms of elementary functions. The axisymmetric motion which is unsteady due to variable velocity of the pipe rotation, and steady state motion with the cylindrical interface have been investigated [2]. The next generalization took into account combined action of the centrifugal force and the transversal gravitational force. Non-stationary variants of the Ostroumov-Birikh solution which describe a plane motion in a horizontal strip, laminar flows of immiscible fluids and unsteady axial convection in a rotating pipe have been presented [3]. Solutions describing the three-dimensional convective flows in a circular tube with the axial temperature gradient have been studied $[3,4]$ and physical interpretation of the obtained solution has been discussed [2,4] (see also [1,3]). The most interesting case appears under zero liquid flow rate. The specific feature of the considered class of flows is the possibility to transfer a passive admixture along pipe for long distance under joint action of the longitudinal thermal gradient and the transversal centrifugal or gravitational force. The three-dimensional exact solution has been constructed to describe two-layer flow in an infinite channel without assumption on axial symmetry of the flow [5]. Components of velocity vector depend on two transversal coordinates. Pressure and temperature have analogous terms. The construction of exact solution of stationary problem is supplemented by numerical investigations including the reduction to corresponding two-dimensional problems.

A sufficiently complete consideration of the concept of "exact solution" of a special form of original convection equations has been introduced [6]. In the classical sense an exact solution is a solution which is expressed in the form of formulae, quadratures, series or special functions. According to [6], a set of exact solutions can be extended by invariant and partially invariant solutions of the equations of hydrodynamics of rank of 1 or 2 . The most of the reviewed results in the present paper satisfy the last condition.

Three-dimensional convective flows in a channel of rectangular cross-section with the longitudinal temperature gradient have been investigated numerically [7-9]. The problem in a horizontal channel of square cross-section with adiabatic lateral walls has been solved with the help of commercial product FLUENT [9]. It has been found that flow pattern depends on the Grashof and Prandtl numbers and on the channel length. Various types of flow symmetry and two ways of the transition to oscillatory regimes with possible symmetry-breaking (fork bifurcation) are possible. Influence of the magnetic field and the effect of geometric sizes of the channel and the Prandtl number on the stability of the flows has been studied ( [8], [7]).

A generalization of the Ostroumov-Birikh solution for description of mixture flows in a cylindrical tube has been developed [10]. The results of a study into nonstationary flows of a 
binary mixture with the thermodiffusion effect were presented. The properties of the invariant solution of the thermodiffusion equations were examined when the surface tension at the interface between two mixtures linearly depends on temperature and concentration. The a priori estimates for all unknown fields of corresponding adjoint initial boundary value problems were proved. The estimations showed exponential convergence of flow characteristics to the stationary values as time increases.

Solution describing liquid flow under the action of co-current or counter-current gas flux and constant longitudinal thermal gradient directed along the cylinder axes has been derived [11]. It was assumed that the considered system is in zero-gravity conditions, free surface is nondeformed, and liquid flow rate is equal to zero. The obtained basic solution is the analogue of the solution that has been constructed beforen [12]. The thermocapillary flows in systems of liquid layers with deformable interface have been also studied [13]. Characteristics of the flows subject to the action of the longitudinal temperature gradient and arising interface deformations under microgravity have been investigated.

\section{Three-dimensional flows with evaporation/condensation}

\subsection{Governing equations and exact solution}

Let us assume that two immiscible fluids (liquid and gas or gas-vapor mixture) fill infinite domains

$$
\begin{gathered}
\Omega_{1}=\left\{(x, y, z):-x_{0}<x<0,0<y<1,-\infty<z<\infty\right\} \\
\left.\Omega_{2}=(x, y, z): 0<x<x^{0}, 0<y<1,-\infty<z<\infty\right\} .
\end{gathered}
$$

Boundaries of the domains $\Omega_{1}, \Omega_{2}$ are the impermeable immovable rigid walls defined by equations $x=-x_{0}, x=x^{0}, y=0, y=1$. The gravitational force vector is $\mathbf{g}=(-g, 0,0)$. The size of the channel in the direction of $O y$ (width of the channel) is chosen as characteristic length. Let $u_{*}$ be the characteristic velocity. It is equal to the velocity of viscous stress relaxation rate, $T_{*}$ is the characteristic temperature drop, $p_{*}=\rho_{1} u_{*}^{2}$ is the characteristic pressure. Liquid from domain $\Omega_{1}$ can evaporate through the thermocapillary interface $\Gamma$. The thermodiffusion and diffusive thermal conductivity effects are taken into account in domain $\Omega_{2}$.

Equations of convection (the Boussinesq approximation of the Navier-Stokes equations) in the non-dimensional form are

$$
\begin{gathered}
\operatorname{div} \mathbf{v}_{i}=0, \quad\left(\mathbf{v}_{i} \cdot \nabla\right) \mathbf{v}_{i}=-\eta_{i}^{p} \nabla p_{i}+\frac{\eta_{i}^{\mathbf{v}}}{R e} \Delta \mathbf{v}_{i}+G_{i} \\
\mathbf{v}_{i} \cdot \nabla T_{i}=\frac{\eta_{i}^{T}}{\operatorname{RePr}}\left(\Delta T_{i}+\underline{\bar{\delta} \Delta C_{s}}\right), \quad i=1,2, \\
\mathbf{v}_{2} \cdot \nabla C_{s}=\frac{1}{P e}\left(\Delta C_{s}+\bar{\alpha} \Delta T_{2}\right) .
\end{gathered}
$$

Parameters and functions with the indices $i=1$ and $i=2$ are related to domains $\Omega_{1}$ (liquid) and $\Omega_{2}$ (gas-vapor mixture), respectively. As before, underlined term in (2.2) and equation (2.3) are used to describe the motion in the upper layer $\Omega_{2}$. The following notations are used: $\eta_{1}^{p}=1$, $\eta_{2}^{p}=1 / \bar{\rho}, \eta_{1}^{\mathbf{v}}=1, \eta_{2}^{\mathbf{v}}=\bar{\nu}, G_{1}=-\mathbf{i}\left(G r / R^{2}\right) T_{1}, G_{2}=-\mathbf{i}\left(\bar{\beta}\left(G r / R e^{2}\right) T_{2}+\gamma\left(G a / R e^{2}\right) C_{s}\right), \mathbf{i}$ is the unit vector of the $O x$ axis, $\eta_{1}^{T}=1, \eta_{2}^{T}=\bar{\chi}$. Here $R e=u_{*} h / \nu_{1}$ is the Reynolds number, $\operatorname{Pr}=\nu_{1} / \chi_{1}$ is the Prandtl number, $G r=\beta_{1} T_{*} g h^{3} / \nu_{1}^{2}$ is the Grashof number, $G a=g h^{3} / \nu_{1}^{2}$ is the Galilei number, $P e=u_{*} h / D$ is the Peclet number (the Peclet diffusion number), $g=|\mathbf{g}|$; $\bar{\rho}=\rho_{2} / \rho_{1}, \bar{\nu}=\nu_{2} / \nu_{1}, \bar{\chi}=\chi_{2} / \chi_{1}, \bar{\beta}=\beta_{2} / \beta_{1}$ are the ratios of the densities, coefficients of 
kinematic viscosity, heat conductivity, thermal expansion, respectively; $\gamma$ is the concentration coefficient of the gas density, $D$ is the coefficient of vapour diffusion in the gas; $\bar{\alpha}=\alpha T_{*}, \bar{\delta}=\delta / T_{*}$, dimensional coefficients $\alpha$ and $\delta$ characterize the Soret and Dufour effects in the gas phase.

We suppose that velocity vector components $\mathbf{v}_{1}=\left(u_{1}, v_{1}, w_{1}\right)$ and $\mathbf{v}_{2}=\left(u_{2}, v_{2}, w_{2}\right)$ depend only on the transversal coordinates $(x, y)$. Temperatures $T_{1}, T_{2}$, pressure (deviation from the hydrostatic pressure) $p_{1}, p_{2}$ and the vapour concentration $C$ include terms $\Theta_{1}, \Theta_{2}, q_{1}, q_{2}, \Phi$. These terms are also dependent on the transversal coordinates $(x, y)$ :

$$
\begin{gathered}
u_{i}=u_{i}(x, y), \quad v_{i}=v_{i}(x, y), \quad w_{i}=w_{i}(x, y), \\
p_{1}=-A \frac{G r}{R e^{2}} x z+q_{1}(x, y), \quad p_{2}=-A \bar{\rho} \bar{\beta} \frac{G r}{R e^{2}} x z+B \bar{\rho} \gamma \frac{G a}{R e^{2}} x z+q_{2}(x, y), \\
T_{i}=-A z+\Theta_{i}(x, y), \quad C_{s}=B z+\Phi(x, y) .
\end{gathered}
$$

Coefficients $A$ and $B$ specify constant longitudinal gradients of the temperature and concentration along the interface. If $A_{*}$ and $B_{*}$ are corresponding non-dimensional gradients, then $A=A_{*} h / T_{*}, B=B_{*} h$. Solution (2.4) is considered as an exact solution in an extended sense [6]. This solution is the partially invariant solution of rank 2 and defect 3 [14].

\subsection{Boundary conditions}

It is assumed that interface is a flat surface.Then equation $x=0$ defines $\Gamma$ in constructing the solution of equations (2.1)-(2.3) in form (2.4). Then one can completely take into account dynamic condition on the interface. It means that there is no need for any simplified form of this condition as it often takes place in hydrodynamic problems. The assumption that interface is non-deformable leads to another boundary condition (see $[15,16]$ ).

Conditions of continuity of the tangential velocities and temperature must be satisfied on the thermocapillary interface $\Gamma$

$$
v_{1}=v_{2}, \quad w_{1}=w_{2}, \quad T_{1}=T_{2} .
$$

The kinematic condition

$$
u_{1}=u_{2}=0
$$

and dynamic condition must be satisfied [5]:

$$
\begin{gathered}
\left(u_{1 y}+v_{1 x}\right)-\bar{\rho} \bar{\nu}\left(u_{2 y}+v_{2 x}\right)=-\frac{M a}{\operatorname{RePr}} \Theta_{1 y}, \quad w_{3 x}-\bar{\rho} \bar{\nu} w_{2 x}=A \frac{M a}{\operatorname{RePr}}, \\
-\operatorname{Re}\left(q_{1}-q_{2}\right)+2\left(u_{1 x}-\bar{\rho} \bar{\nu} u_{2 x}\right)=0 .
\end{gathered}
$$

Relations (2.7) and (2.8) are projections of the dynamic condition on the tangential and normal to $\Gamma$ vectors, respectively. The diffusive mass flux of the evaporating liquid and the mass balance equation are taken into consideration. Then we have heat transfer condition of the form (see Part I)

$$
T_{1 x}-\bar{\kappa} T_{2 x}-\bar{\delta} \bar{\kappa} C_{s x}=-\bar{L} M, \quad M=-\left(C_{s x}+\bar{\alpha} T_{2 x}\right), \quad \bar{L}=L D \rho_{2} /\left(\kappa_{1} T_{*}\right),
$$

where $\bar{\kappa}=\kappa_{2} / \kappa_{1}$ is the ratio of the thermal conductivity coefficients, characteristic mass flow rate of evaporation is chosen equal to $M_{*}=D \rho_{2} / h$. The linearised equation for the saturated vapour concentration on $\Gamma$ is written in the dimensionless form as follows:

$$
\left.C_{s}\right|_{x=0}=C_{*}\left(1+\bar{\varepsilon}\left(T_{2}-T_{0}\right)\right), \quad \bar{\varepsilon}=\varepsilon T_{*} .
$$


The following notations are introduced: $M a=\sigma_{T} T_{*} h /\left(\rho_{1} \nu_{1} \chi_{1}\right)$ is the Marangoni number, $C a=\rho_{1} \nu_{1} u_{*} / \sigma_{0}$ is the capillary number, $H$ is the surface mean curvature (here $H=0$ ). Condition (2.8) with zero right-hand side can be considered as the first order approximation to equation (2.8) in the case of small capillary number $\mathrm{Ca}$. As in the Part I the linear dependence of the surface tension coefficient on temperature is assumed $\left(\sigma=\sigma_{0}-\sigma_{T}\left(T-T_{0}\right)\right)$.

When vapour flux on the upper and lateral rigid boundaries is absent we have [17]:

$$
x=x^{0}: \quad C_{s x}=0 ; \quad y=0, y=y^{0}: \quad C_{s y}=0 .
$$

No-slip conditions for velocity vectors on the channel walls are assumed

$$
x=-x_{0}, x=x^{0}, y=0, y=y^{0}: \quad \mathbf{v}_{i}=0 .
$$

Lateral walls are thermally insulated:

$$
\begin{gathered}
x=-x_{0}: \quad T_{1 x}=0 ; \quad x=x^{0}: \quad T_{2 x}=0 \\
y=0: \quad T_{1 y}=0, T_{2 y}=0 ; \quad y=y^{0}: \quad T_{1 y}=0, T_{2 y}=0 .
\end{gathered}
$$

Let us note that conditions (2.11), (2.13) mean that full heat flux with respect to the Dufour effect is equal to zero on the fixed walls

$$
\frac{\partial T_{2}}{\partial n}+\bar{\delta} \frac{\partial C_{s}}{\partial n}=0
$$

and full mass flux with respect to the Soret effect is also equal to zero on the fixed walls

$$
\frac{\partial C_{s}}{\partial n}+\bar{\alpha} \frac{\partial T_{2}}{\partial n}=0 .
$$

In the case of zero vapour concentration on the upper and lateral rigid boundaries of the channel

$$
x=x^{0}: \quad C_{s}=0 ; \quad y=0, y=y^{0}: \quad C_{s}=0
$$

and condition of type (2.14) should be set on the same walls.

\section{Numerical study}

The analytical technique to model stationary three-dimensional convection of the immiscible fluids with evaporation at the interface is supplemented by the numerical studies $[5,17]$. Derivation of the stationary solutions of type (2.4) for the two-layer convective fluid flows is reduced to the two-dimensional problems for the functions $w_{1}(x, y)$ and $w_{2}(x, y), \Theta_{1}(x, y)$, $\Theta_{2}(x, y)$ and $\Phi(x, y)$, and for the stream functions $\psi_{1}(x, y), \psi_{2}(x, y)$ and vorticity $\omega_{1}(x, y)$, $\omega_{2}(x, y)$ instead of the transverse components $u_{i}, v_{i}$ of the velocity vectors. As usual we put $u_{i}=\left(\psi_{i}\right)_{y}, v_{i}=-\left(\psi_{i}\right)_{x}, \omega_{i}=v_{i x}-u_{i y}(i=1,2)$. Functions $\psi_{i}, \omega_{i}$ are found as the solutions of the following systems of equations

$$
\Delta \psi_{i}=-\omega_{i}, \quad u_{i} \omega_{i x}+v_{i} \omega_{i y}=\frac{\eta_{i}^{\omega}}{R e} \Delta \omega_{i}+\widetilde{G}_{i}, \quad i=1,2
$$

All two-dimensional problems are solved in the domains $\widetilde{\Omega}_{i}$ which are the cross-sections of the domains $\Omega_{i}$ by the plane $z=z_{0}\left(z_{0}\right.$ is an arbitrary constant), i.e.

$$
\widetilde{\Omega}_{1}=\left\{(x, y):-x_{0}<x<0,0<y<1\right\}, \quad \widetilde{\Omega}_{2}=\left\{(x, y): 0<x<x^{0}, 0<y<1\right\} .
$$


The full boundary $\partial \widetilde{\Omega}_{1}$ of the cross-section $\widetilde{\Omega}_{1}$ is defined by relations $x=-x_{0}, x=0, y=0$, $y=1$; the full boundary $\partial \widetilde{\Omega}_{2}$ of the cross-section $\widetilde{\Omega}_{2}$ is defined by relations $x=x^{0}, x=0, y=0$, $y=1$. The interface $\widetilde{\Gamma}$ is the intersection of the interface $\Gamma$ and cross-sections $\widetilde{\Omega}_{i}$ and defined by relation $x=0$. In the second equation (3.1) coefficient $\eta_{1}^{\omega}=1$ and $\eta_{2}^{\omega}=\bar{\nu} ; \widetilde{G}_{1}=-\left(G r / R e^{2}\right) \Theta_{1 y}$, $\widetilde{G}_{2}=-\left(\bar{\beta}\left(G r / R e^{2}\right) \Theta_{2 y}+\gamma\left(G a / R e^{2}\right) \Phi y\right)$.

The free boundary conditions are reformulated in terms of $\psi_{I}-\omega_{i}$ (for the case of curved free boundary see [18-20]). The kinematic condition (2.6) has the form

$$
\psi_{1 y}=\psi_{2 y}=0
$$

Then $\psi_{1}=\psi_{2}=$ const on the interface $\widetilde{\Gamma}$ (or at $x=0$ ). Without loss of generality we can set

$$
\psi_{1}=\psi_{2}=0
$$

Condition of equality of the tangential velocities (2.5) at the interface $\widetilde{\Gamma}$ leads to the relation

$$
\psi_{1 x}=\psi_{2 x}
$$

The first dynamic boundary condition (2.7) can be written in the form:

$$
\omega_{1}-\bar{\rho} \bar{\nu} \omega_{2}=-\frac{M a}{R e P r} \Theta_{1 y}
$$

Normal component of dynamic condition (2.8) written with the help of (3.2) is differentiated along the interface $\widetilde{\Gamma}$ (with respect to $y$ in the present consideration). Then, using equations (2.1) for $q_{i y}$ and assuming that we can change the order of differentiation only to get the terms with $\omega_{i x}$, condition $(2.8)$ is rewritten as

$$
2\left(\psi_{1 y x}-\bar{\rho} \bar{\nu} \psi_{2 y x}\right)_{y}=(\bar{\rho}-1) \operatorname{Re} \psi_{1 x} \psi_{1 x y}+\omega_{1 x}-\bar{\rho} \bar{\nu} \omega_{2 x} .
$$

The dynamic conditions $(3.5),(3.6)$ are valid on $\widetilde{\Gamma}$. For a curved free boundary similar results have been obtained $[18,19]$.

Taking into account (3.3), no-slip conditions (2.12) can be written in terms of stream function for each part of the boundary $\widetilde{\Omega}_{i}$ (i.e. at $x=-x_{0}, x=x^{0}, y=0, y=1$ )/ Then we have the following boundary conditions:

$$
\left.\psi_{i}\right|_{\partial \widetilde{\Omega}_{i}}=0,\left.\quad \psi_{1 x}\right|_{x=-x_{0}}=0,\left.\quad \psi_{2 x}\right|_{x=x^{0}}=0,\left.\quad \psi_{i y}\right|_{y=0, y=1}=0 .
$$

Numerical algorithm for solving two-dimensional problems is based on a finite-difference scheme known as the alternating direction method [21,22]. The general scheme of solving the stationary problem is an iterative process with consistent realization of the following stages:

(I). Let the velocity components $u_{i}, v_{i}$ be found. With given $u_{i}, v_{i}$ the problems for the third components of the velocity vectors $w_{i}$ are solved numerically.

(II). To obtain the unknown components $\Theta_{i}$ and $\Phi$ of temperature and vapor concentration distributions the systems of corresponding equations and boundary conditions at the interface and rigid walls are solved.

(III). When $\Theta_{i}$ and $\Phi$ are known we solve numerically the systems of the equations for the stream functions and vorticity $\psi_{i}, \omega_{i}(i=1,2)$.

(IV). The transverse components $u_{i}, v_{i}$ of the velocity vectors are determined from the stream functions and then we return to stage (I). 


\section{Pattern of the three-dimensional flows with evaporation}

The numerical studies are carried out for a liquid - gas system like "ethanol-nitrogen". Values of the physical parameters of the liquid-gas system are given in Tab. 1. It is assumed that the non-dimensional Soret and Dufour parameters are equal to $\bar{\alpha}=10^{-3}$ and $\bar{\delta}=10^{-5}$. Computations are performed with the the Grashof number $G r=\{47000,470\}$ which correspond to the conditions of normal $\left(g=g_{0}=9.81 \mathrm{~m} / \mathrm{s}^{2}\right)$ and low gravity $\left(g=g_{0} \cdot 10^{-2} \mathrm{~m} / \mathrm{s}^{2}\right)$ and to the characteristic temperature drop $T_{*}=10 \mathrm{~K}$; the characteristic length is equal to $h=10^{-2} \mathrm{~m}$. In the numerical experiments the non-dimensional longitudinal temperature gradient $A$ has the values $A=0.4$ and $A=0.1$. It corresponds to the dimensional values $A_{*}=400 \mathrm{~K} / \mathrm{m}$ and $A_{*}=100 \mathrm{~K} / \mathrm{m}$. The non-dimensional liquid layer thickness $x_{0}$ has values $x_{0}=0.1$ and $x_{0}=0.25$ that correspond to the dimensional values $x_{0}^{*}=1 \mathrm{~mm}$ and and $x_{0}^{*}=2.5 \mathrm{~mm}$, respectively. Gas layer thickness $x^{0}$ is chosen as $x^{0}=0.5$ that is in accord with the dimensional value $x_{*}^{0}=5 \mathrm{~mm}$. The coefficient $B$ is defined with help of $(2.10): B=-C_{*} \bar{\varepsilon} A$.

Table 1. Values of the physical parameters for the system "ethanol-nitrogen"

\begin{tabular}{|l|c|c|}
\hline Parameter $/$ Medium & Ethanol & Nitrogen \\
\hline Density $\rho, \mathrm{kg} / \mathrm{m}^{3}$ & $0.79 \cdot 10^{3}$ & 1.2 \\
\hline Kinematic viscosity $\nu, \mathrm{m}^{2} / \mathrm{s}$ & $0.15 \cdot 10^{-5}$ & $0.15 \cdot 10^{-4}$ \\
\hline Coefficient of thermal expansion $\beta, K^{-1}$ & $0.108 \cdot 10^{-2}$ & $3.67 \cdot 10^{-3}$ \\
\hline Coefficient of thermal conductivity $\kappa, \mathrm{W} /(\mathrm{m} \cdot \mathrm{K})$ & 0.1672 & 0.02717 \\
\hline Coefficient of thermal diffusivity $\chi, \mathrm{m}^{2} / \mathrm{s}$ & $0.89 \cdot 10^{-7}$ & $0.3 \cdot 10^{-4}$ \\
\hline Temperature coefficient of surface tension $\sigma_{T}, \mathrm{~N} /(\mathrm{m} \cdot \mathrm{K})$ & $0.8 \cdot 10^{-4}$ & \\
\hline Latent heat of evaporation $L,(\mathrm{~W} \cdot \mathrm{s}) / \mathrm{kg}$ & $8.55 \cdot 10^{5}$ & \\
\hline Molar mass of evaporating liquid $\mu, \mathrm{kg} / \mathrm{mol}$ & 0.046 & \\
\hline Coefficient of diffusion $D, \mathrm{~m}^{2} / \mathrm{s}$ & & $0.135 \cdot 10^{-4}$ \\
\hline Coefficient of concentration expansion $\gamma$ & & -0.62 \\
\hline Saturated vapor concentration $C_{*}$ & & 0.1 \\
\hline
\end{tabular}

Figs. 1-4 present possible three-dimensional flow patterns in the system with evaporation/condensation at various values of the longitudinal temperature gradient $A$ and liquid layer thickness $x_{0}$ under the standard conditions with $G r=47000$. The exact solution (2.4) describes formation of the longitudinal thermocapillary rolls (see Fig. 1, 3(a)). The structures appear due to the thermocapillary effect which causes liquid spreading on the surface from the hot pole to cold one. Upon that in the lower layer the stable temperature stratification with the characteristic thermal "roll" along the channel axes is formed (Fig. 2(a)) and liquid motion is induced mainly by the Marangoni effect even under weak thermal load. Near the lateral walls the liquid moves down due to continuity. It results in formation of vortex "cores" in corners. Unstable temperature stratification in the upper layer leads to the convective motion of a gas that is more intensive than in the liquid.

At small temperature gradients the flow pattern has distinct symmetrical two-vortex structure in each phase (Fig. 1(a), 3(a)). When the longitudinal temperature gradient increases the thermocapillary effect becomes more intensive, and the thermocapillary rolls fall into smaller shafts (Fig. 1(b)). The resulting motion has translational-rotational character, and liquid particles move along the axes of each roll from domain with higher temperature to cold region. Several thermal "spots" arises on the interface due to condensation (upon that $M<0$, Fig. 2(b)). Let us 


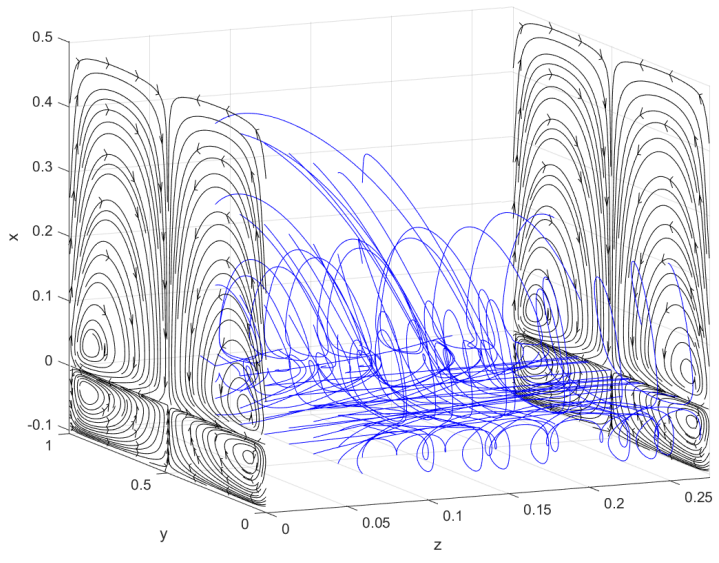

(a)

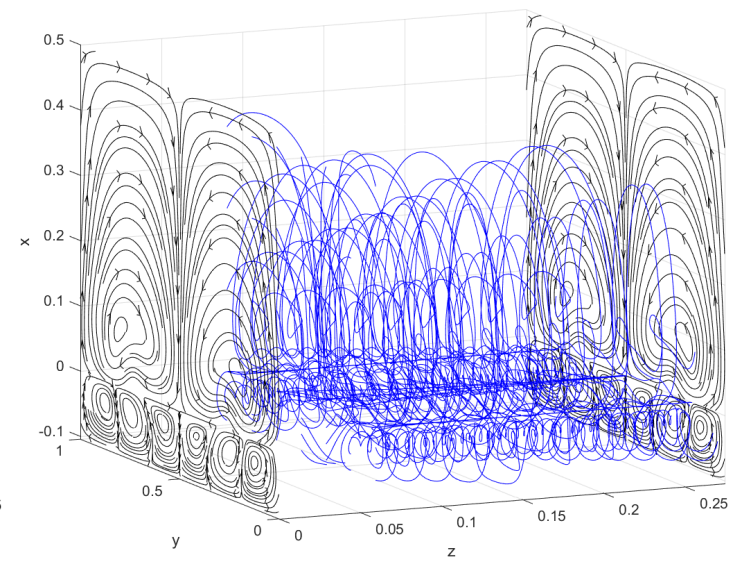

(b)

Fig. 1. Streamlines and trajectories in the system with evaporation at $G r=47000, x_{0}=0.1$ : (a) $-A=0.1 ;(b)-A=0.4$

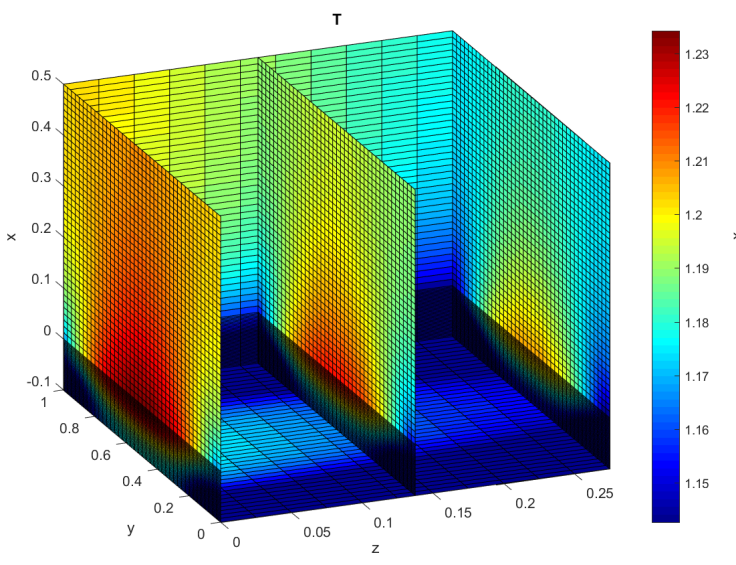

(a)

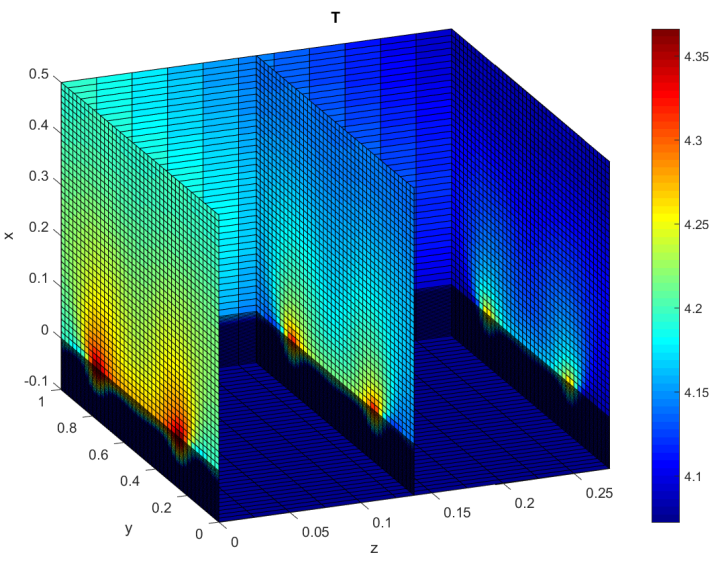

(b)

Fig. 2. Temperature distribution in the system with evaporation at $G r=47000, x_{0}=0.1:(a)-$ $A=0.1 ;(b)-A=0.4$

note that in thin layers the thermocapillary effect dominates over the evaporation/condensation processes. Heat transfer induced by the Marangoni effect is more intensive than temperature variations caused by evaporation at small $A$ or condensation at large gradients.

When the liquid layer thickness increases at weak thermal load qualitative picture of the flows does not changed. Such liquid layer is less sensitive to the thermocapillary effect and evaporation. Two-vortex patterns in each medium are formed but motion becomes predominantly translational as liquid is driven by the co-current gas flux (Fig. 3(a)). The thermocapillary shafts arise and their formation is caused by the liquid spreading from central hot temperature "roll" to the lateral walls (Fig. 4(a)). Growth of $A$ results in essential alteration of the flow topology and temperature distribution. At quite large value of $A$ condensation occurs in the system $(M<0$, temperature decreases along $O z$ axes when $A$ increases, see form of exact solution (2.4)). It provides an energy inflow in liquid phase. The supply of heat is more significant in comparison with the cooling induced by the applied temperature gradient. In liquid layer the thermal "fingers" appear (Fig. 4(b)). Hydrodynamical characteristics are also altered. Basic 


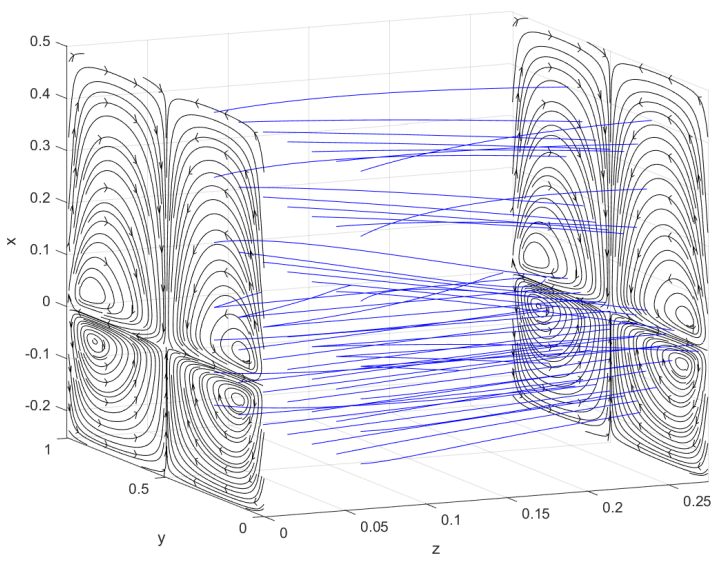

(a)

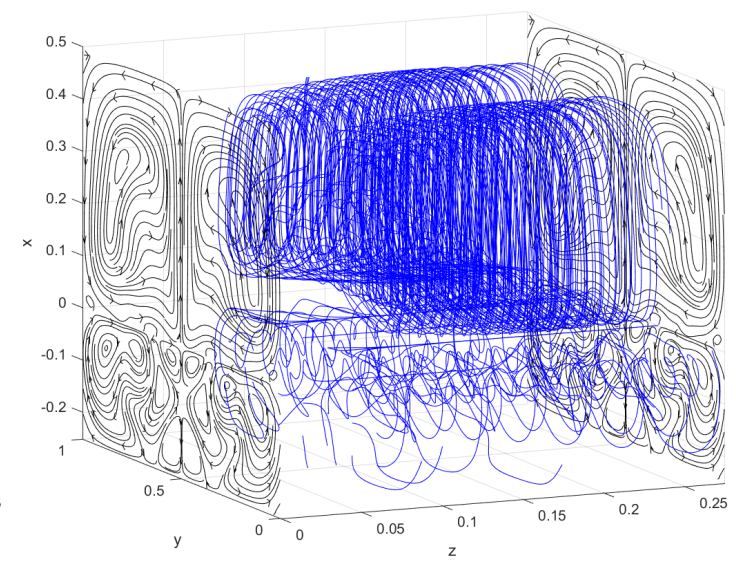

(b)

Fig. 3. Streamlines and trajectories in the system with evaporation at $G r=47000, x_{0}=0.25$ : (a) $-A=0.1 ;(b)-A=0.4$

vortexes are distorted, and deformations are accompanied by appearance of additional cores in the big vortexes and of additional small swirls near the interface (Fig. 3(b)). At large $A$ the thermocapillary effect results in more intensive motion on the interface and, hence, within liquid layer.

Figs. 5-8 present possible three dimensional flow patterns in the system with evaporation/condensation at different values of the longitudinal temperature gradient $A$ and liquid layer thickness $x_{0}$ under the microgravity conditions with $G r=470$. For system with thin liquid layer (Fig. 5) the thermocapillary mechanism is the basic one and it defines the structure of the flows.

The influence of the thermal stratification in weak gravitational field becomes less substantial and intensity of the translational-rotational motion is maintained by the Marangoni effect. If values of $A$ are small then temperature variations due to thermal load is more significant in comparison with the changes generated by evaporation. At large $A$ central thermal "shaft" is split into two "rolls" (Fig. 6(b)). Liquid moves from hot regions to cold zones on the interface and again the number of the longitudinal thermocapillary structures increases (Fig. 5(b)). We observe formation of the symmetrical six-vortex cellular pattern with two small "central" thermocapillary structures.

In the system with weak thermal load increasing the thickness of the lower layer does not lead to alteration of the flow pattern, and only quantitative variations in the temperature distribution take place (Fig. 7(a), 8(a)).

At large $A$ two thermoclines arise in the liquid layer (Fig. 8(b)). Condensation provides heat inflow from gas to liquid phase. Cold zone in the central part of the channel is formed due to the thermocapillary effect which forces liquid to move from hot near-wall domains to the channel axes. Upon that in this zone two small swirls with opposite circulation are formed. These vortices have convective nature. Big deformed symmetrical vortices have two cores. Angled parts of these vortices are originated by the Marangoni effect, and motion in central parts of these swirls occurs due to continuity. Close to the bottom of the channel two-core additional vortices appear (Fig. 7(b)).

Let us note that at large $A$ transformation of two-vortex structures occurs in the gas-vapor layer. In most considered configurations the unstable temperature stratification is formed in 


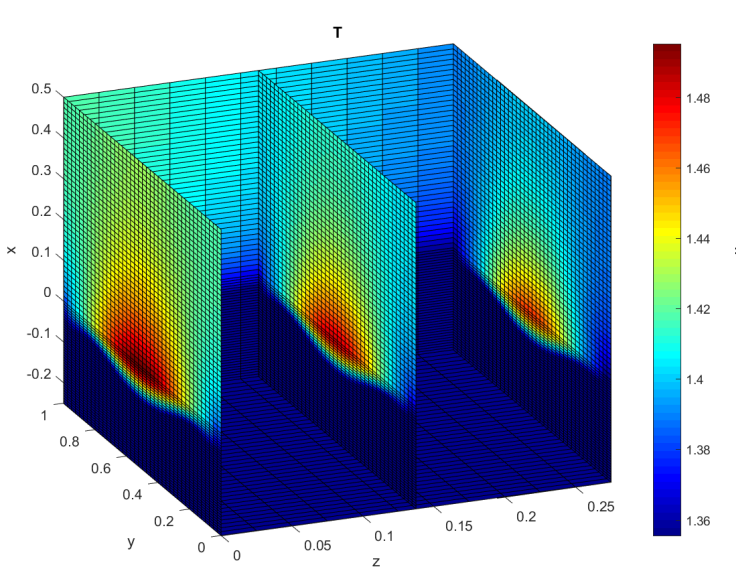

(a)

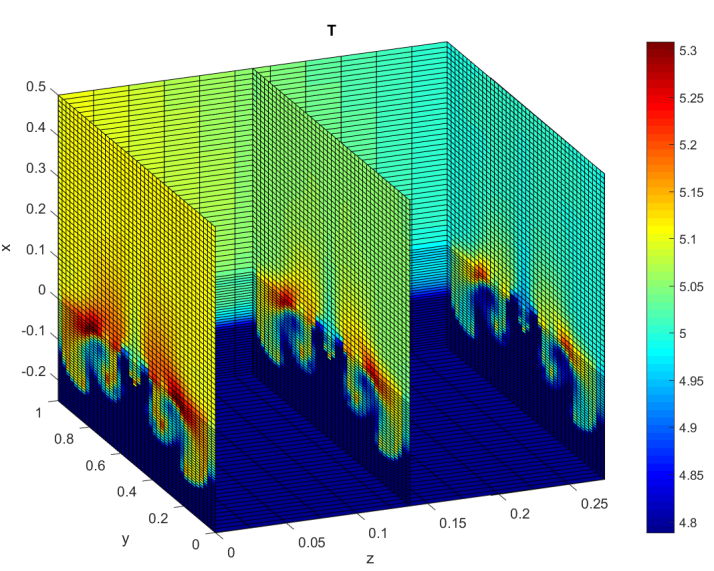

(b)

Fig. 4. Temperature distribution in the system with evaporation at $G r=47000, x_{0}=0.25$ : (a) $-A=0.1 ;(b)-A=0.4$

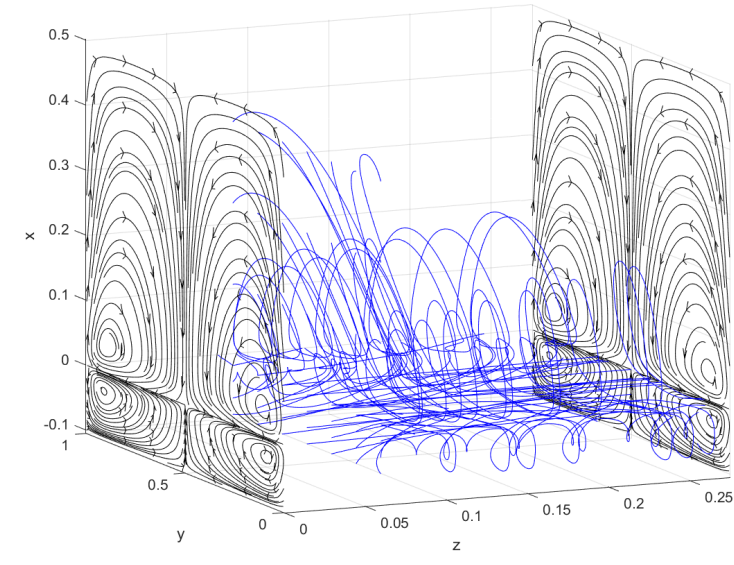

(a)

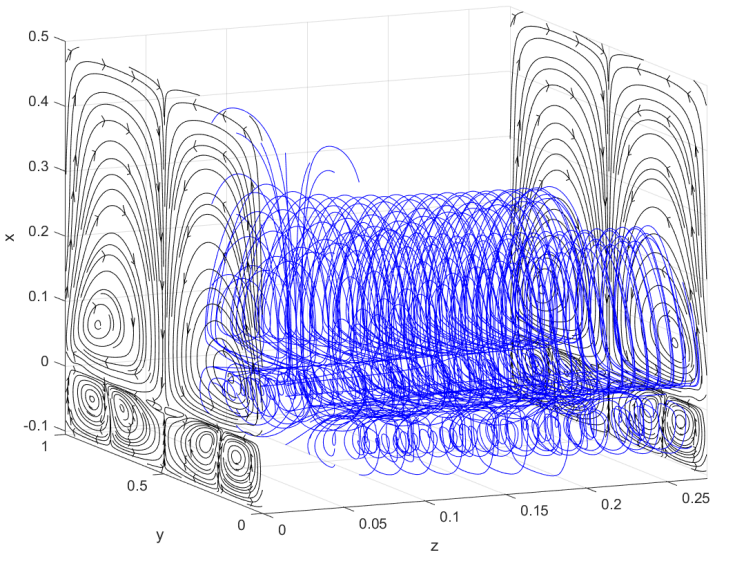

(b)

Fig. 5. Streamlines and trajectories in the system with evaporation at $G r=470, x_{0}=0.1:(a)-$ $A=0.1 ;(b)-A=0.4$

the upper layer. Therefore, observed structures are the convective cells. Alteration of the hydrodynamic field is generated by splitting the central thermal "roll" into the several "shafts" (Fig. 2(b), 4(b), 6(b)) or by appearance cold thermal "fingers" (Fig. 8(b)). Formation of the latter is induced by intensive condensation.

Thus, considered exact solution (2.4) describes various regimes of convective flows accompanied by evaporation/condensation. Results obtained on the basis of this solution qualitatively agree with the known experimental data. In particular, it describes appearance of the thermocapillary rolls and thermal "fingers", cellular and thermocapillary structures. In general, with the help of an exact solution we can evaluate an impact of particular factors and clarify character of each mechanism. Therefore, exact solution is the invaluable instrument of studying such multi-parameter problem as evaporative convection.

The work was supported by Russian Science Foundation (project 15-19-20049). 


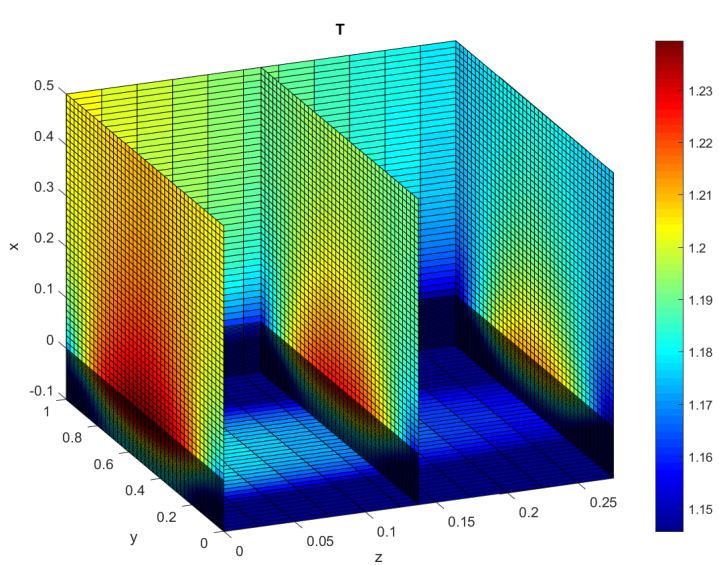

(a)

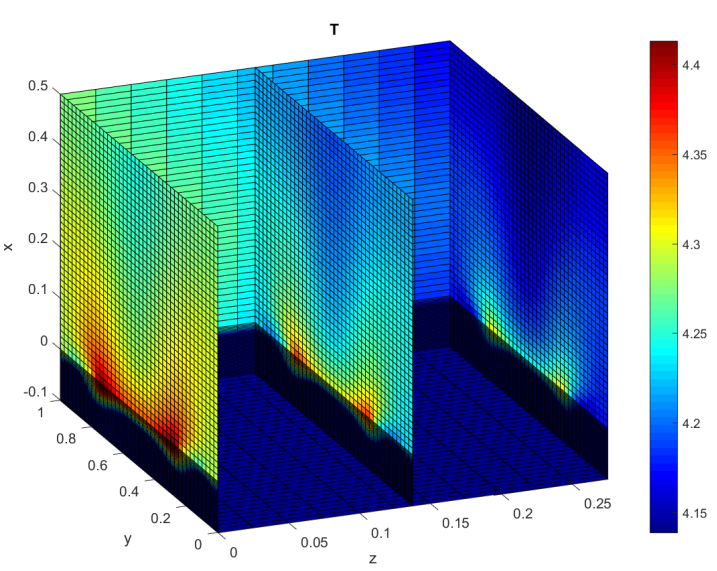

(b)

Fig. 6. Temperature distribution in the system with evaporation at $G r=470, x_{0}=0.1:(a)-$ $A=0.1 ;(b)-A=0.4$

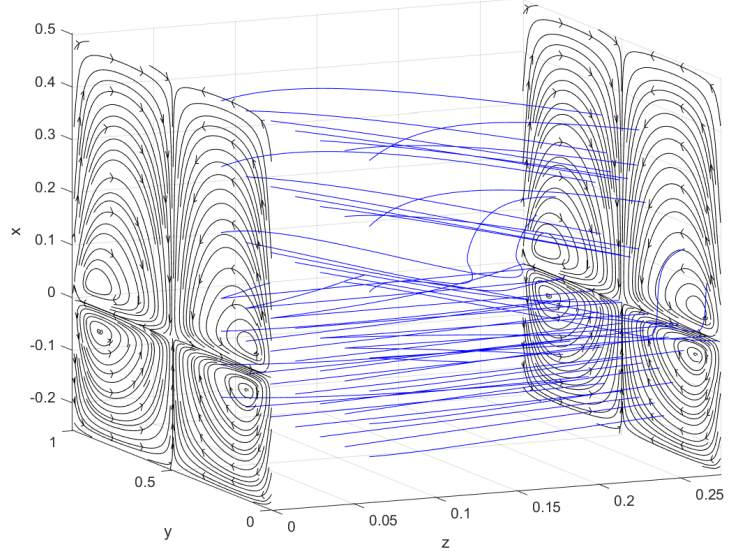

(a)

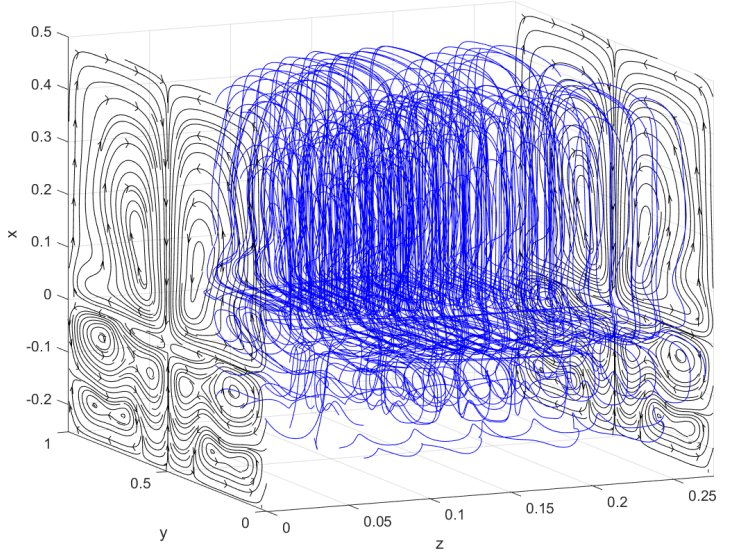

(b)

Fig. 7. Streamlines and trajectories in the system with evaporation at $G r=470, x_{0}=0.25$ : (a) $-A=0.1 ;(b)-A=0.4$

\section{References}

[1] V.V.Pukhnachov, Group-theoretical nature of the Birich's solution and its generalizations, Book of Proc. "Symmetry and differential equations", Krasnoyarsk, 2000, 180-183 (in Russian).

[2] R.V.Birikh, V.V.Pukhnachev, An axial convective flow in a rotating tube with a longitudinal temperature gradient, Dokl. Phys., 56(2011), no. 1, 47-52.

[3] V.V.Pukhnachov, Nonstationary analogues of Birikh solutions, Izvestiya Altaiskogo gosudarstvennogo universiteta, 69(2011), no. 1/2, 62-69 (in Russian).

[4] V.V.Pukhnachov, Convective flows in the long tubes, Bulleten Nizhnyi Novgorod Univer., Nizhnyi Novgorod, 4(2011), no. 5, 2446-2448. 


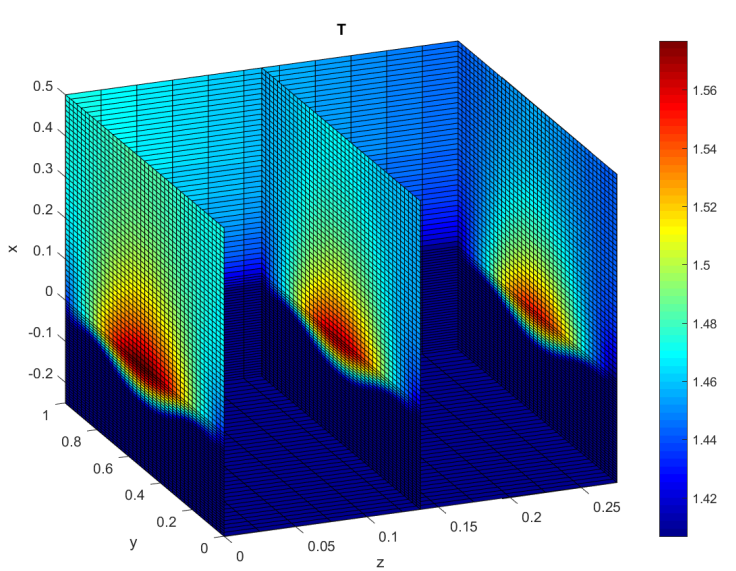

(a)

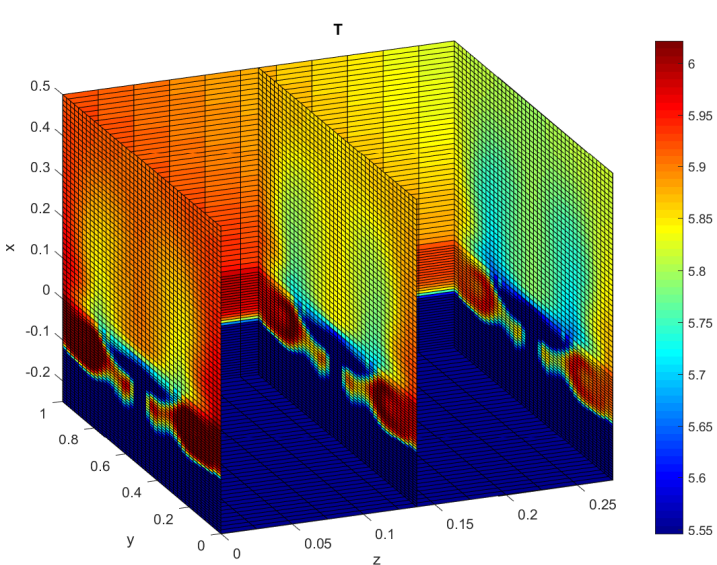

(b)

Fig. 8. Temperature distribuyion in the system with evaporation at $G r=470, x_{0}=0.25:(a)-$ $A=0.1 ;(b)-A=0.4$

[5] O.N.Goncharova, O.A.Kabov, V.V.Pukhnachov, Solutions of special type describing the three dimensional thermocapillary flows with an interface, Int. J. Heat Mass Transfer, 55(2012), no. 4, 715-725.

[6] V.K.Andreev, O.V.Kaptsov, V.V.Pukhnachov, A.A.Rodionov, Applications of group theoretical methods in hydrodynamics, Kluwer Academic Publ., Dordrecht, Boston, London, 1998.

[7] T.P.Lyubimova, D.V.Lyubimov, V.A.Morozov, R.V.Scurdin, H.Ben Hadid, D.Henry, Stability of convection in a horizontal channel subjected to a longitudinal temperature gradient, Part 1, Effect of aspect ratio and Prandtl number, J. Fluid Mech., 635(2009), 275-295.

[8] D.V.Lyubimov, T.P.Lyubimova, A.B.Perminov, D.Henry, H.Ben Hadid, Stability of convection in a horizontal channel subjected to a longitudinal temperature gradient, Part 2, Effect of aspect ratio and Prandtl number, J. Fluid Mech., 635(2009), 297-319.

[9] T.P.Lyubimova, D.A.Nikitin, Three-dimensional advective flows in a horizontal square crosssection cylinder with adiabatic lateral walls, Computational continuum mechanics, 4(2011), no. $2,72-81$.

[10] V.K.Andreev, N.L.Sobachkina, Motion of Binary Mixture in Plane and Cylindrical Domains, Sib. Federal Uni., Krasnoyarsk, 2012 (in Russian).

[11] I.I.Ryzhkov, V.M.Shevtsova, Thermocapillary instabilities in liquid columns under co-and counter-current gas flows, Int. J. Heat Mass Transfer, 55 (2012), no. 4, 1236-1245.

[12] O.Goncharova, O.Kabov, Gas flow and thermocapillary effects on fluid flow dynamics in a horizontal layer, Microgravity Sci. Technol., 21(2009), no. 1, S129-S137.

[13] V.A.Briskman, T.P.Lyubimova, A.A.Nepomnyaschy, A.L.Zuev, Thermocapillary flows and deformations of the surface in the systems of fluid layers with the longirudinal tempperature gradient in microgravity, Microgravity Sci. Technol., IV(1991), no. 2, 98-99. 
[14] V.K.Andreev, The Birikh solution of the convection equations and some its generalizations, Pre-Print no. 1-10, Krasnoyarsk, ICM SB RAS, 2010 (in Russian).

[15] R.Kh.Zeytounian, The Benard-Marangoni thermocapillary-instability problem, Usp. Phys. Nauk, 168(1998), no. 3, 259-286.

[16] A.A.Nepomnyashchy, M.G.Velarde, P. Colinet, Interfacial phenomena and convection, Boca Raton: Chapman \& Hall/CRC, 2002.

[17] O.N.Goncharova, O.A.Kabov, Investigation of the two-layer fluid flows with evaporation at interface on the basis of the exact solutions of the 3D problems of convection, Journal of Physics: Conference Series, 754(2016), 032008.

[18] V.V.Pukhnachov, Viscous fluid flow with free boundaries, Novosibirsk. Gos. Univ., Novosibirsk, 1989 (in Russian).

[19] W.Doerfler, O.Goncharova, D.Kroener, Fluid flow with dynamic contact angle: numerical simulation, ZAMM, 82 (2002), no. 3, 167-176.

[20] A.S.Ovcharova, Method of calculating steady-state flows of a viscous fluid with free boundary in vortex-stream function variables, J. Appl. Mech. Techn. Phys., 39(1998), no. 2, $211-219$.

[21] N.N.Yanenko, The method of fractional steps: the solution of problems of mathematical physics of several variables, Springer Verl., Berlin, Heidelberg, New York, 1971.

[22] V.K.Andreev, Yu.A.Gaponenko, O.N. Goncharova, V.V. Pukhnachov, Mathematical models of convection, De Gruyter, Berlin/Boston, 2012. 


\section{Анализ точного решения задачи испарительной конвекции (обзор). Часть II. Трехмерные течения}

Виктория Б. Бекежанова

Институт вычислительного моделирования СО РАН

Академгородок, 50/44, Красноярск, 660036

Сибирский федеральный университет

Свободный, 79, Красноярск, 660041

Россия

Ольга Н. Гончарова

Институт теплофизики им. Кутателадзе СО РАН

Лаврентьева, 1, Новосибирск, 630090

Алтайский государственный университет

Ленина 61, Барнаул, 656015

Россия

Илья А. Шефер

Институт математики и фундаментальной информатики

Сибирский федеральный университет

Свободный, 79, Красноярск, 660041

Россия

В настоящей работе представлен обзор точных решений трехмерных задач конвекиии для моделирования двухслойных конвективных течений с испарением на термокапиллярной границе раздела.

Ключевые слова: испарительная конвекция, точное решение, трехмерные течения, термокапиллярная граница раздела. 